\title{
A Case of Arterioportal Fistula Leading to Non-cirrhotic Portal Hypertension
}

\author{
Sheela S. Reddy ${ }^{1}$, Raja K. Dhanekula ${ }^{1}$, Warren Maley ${ }^{2}$, Colette Shaw ${ }^{3}$, Jesse M. Civan ${ }^{1}$, David A. Sass ${ }^{1}$, \\ Jonathan M. Fenkel ${ }^{1}$, Susan Shamimi-Noori ${ }^{3}$, Dina Halegoua-Demarzio ${ }^{1}$ \\ 1 Division of Gastroenterology and Hepatology, Thomas Jefferson University Hospital \\ 2 Division of Transplant Surgery, Thomas Jefferson University Hospital \\ ${ }^{3}$ Department of Radiology, Thomas Jefferson University Hospital
}

\section{Background}

Arterioportal fistula (APF) is a rare cause of non-cirrhotic portal hypertension.

This is a case of a hepatic artery-portal vein fistula presenting with recurrent variceal hemorrhage and ascites in a patient with no known liver disease.

\section{Methods}

Case report and literature review

\section{Case}

61-year-old woman with history of aplastic anemia on cyclosporine, remote colon cancer s/p sigmoid resection with work up including a percutaneous liver biopsy to evaluate a benign liver lesion as a possible metastasis about 10 years prior to this current presentation.

She initially presented to an outside hospital with hematemesis, as well as a new onset of increasing abdominal girth and lower extremity edema

EGD showed bleeding esophageal varices that were successfully band ligated. Over the next 6-weeks, she required multiple EGDs with band ligation for recurrent variceal bleeding.

She also had evidence of ascites and diagnostic paracentesis demonstrated a high serum ascites albumin gradient (SAAG) of 3.1. Total Protein in ascitic fluid was 2.6

She was ultimately referred to our center for evaluation of her candidacy for a possible transjugular intrahepatic portosystemic shunt (TIPS).

CT scan was notable for right hepatic lobe atrophy, with marked enlargement of the right hepatic artery and portal vein.

Subsequent MRI identified a right hepatic lobe arteriovenous malformation between the right hepatic artery and portal vein with evidence of portal hypertension.

\section{Case (continued)}

- Transjugular liver biopsy with pressures was notable for a hepatic venous pressure gradient (HVPG) of $7.5 \mathrm{mmHg}$.

- Liver biopsy showed perisinusoidal fibrosis in zone three without cirrhosis, and intimal hyperplasia of portal vein (arteriolization) consistent with arterioportal fistula (Figure 1).

- Rather than pursuing TIPS, we elected to perform coil embolization of the two right hepatic arteries supplying the fistula (Figure 2).

Despite technical success, a small fistula persisted and repeat embolizations were required. She has not needed paracentesis nor has any bleeding during the 1 year follow-up.

Figure 1: Liver biopsy showing arteriolization of the portal vein

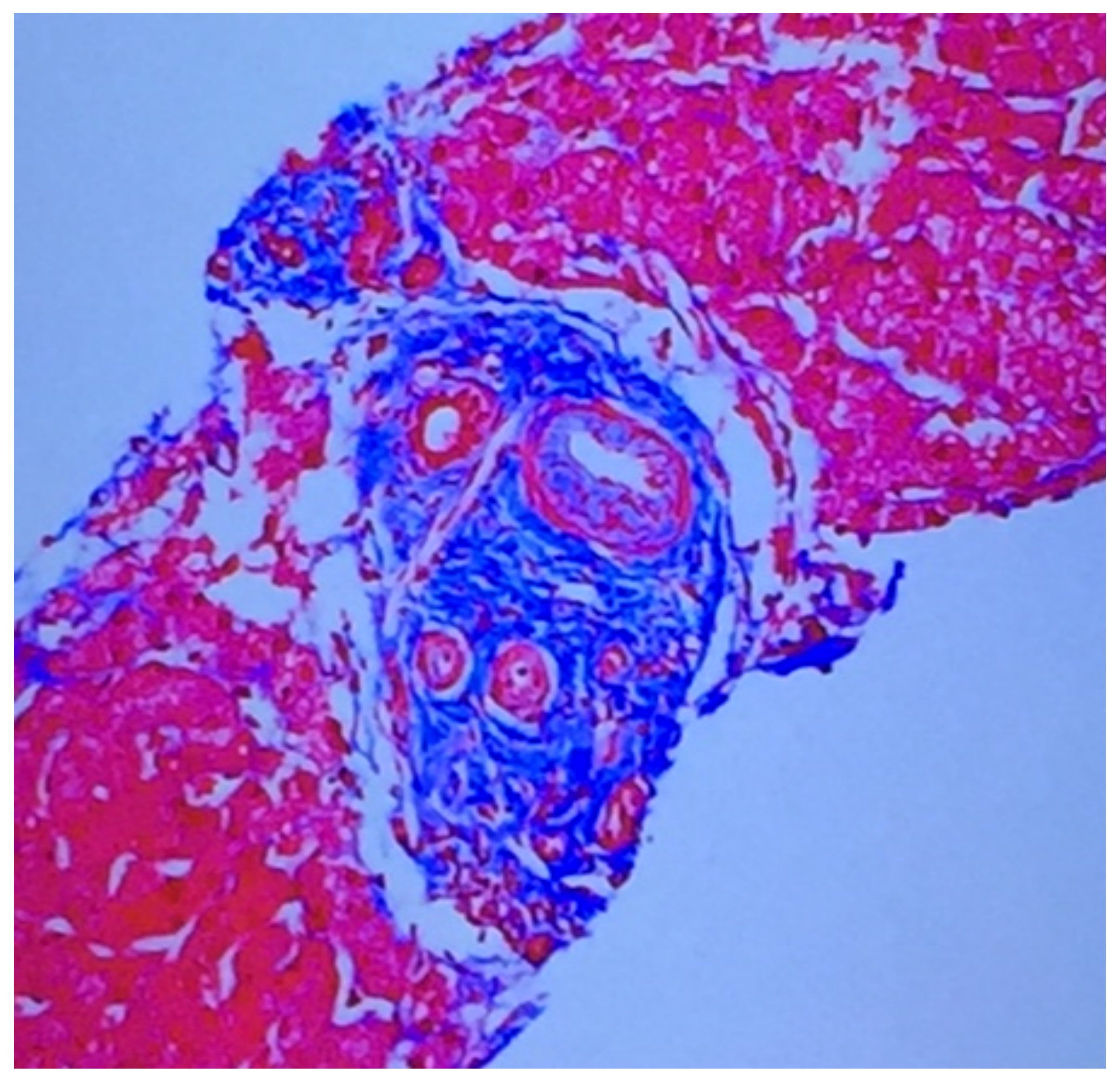

Figure 2: Hepatic artery-portal vein fistula before and after coil embolization

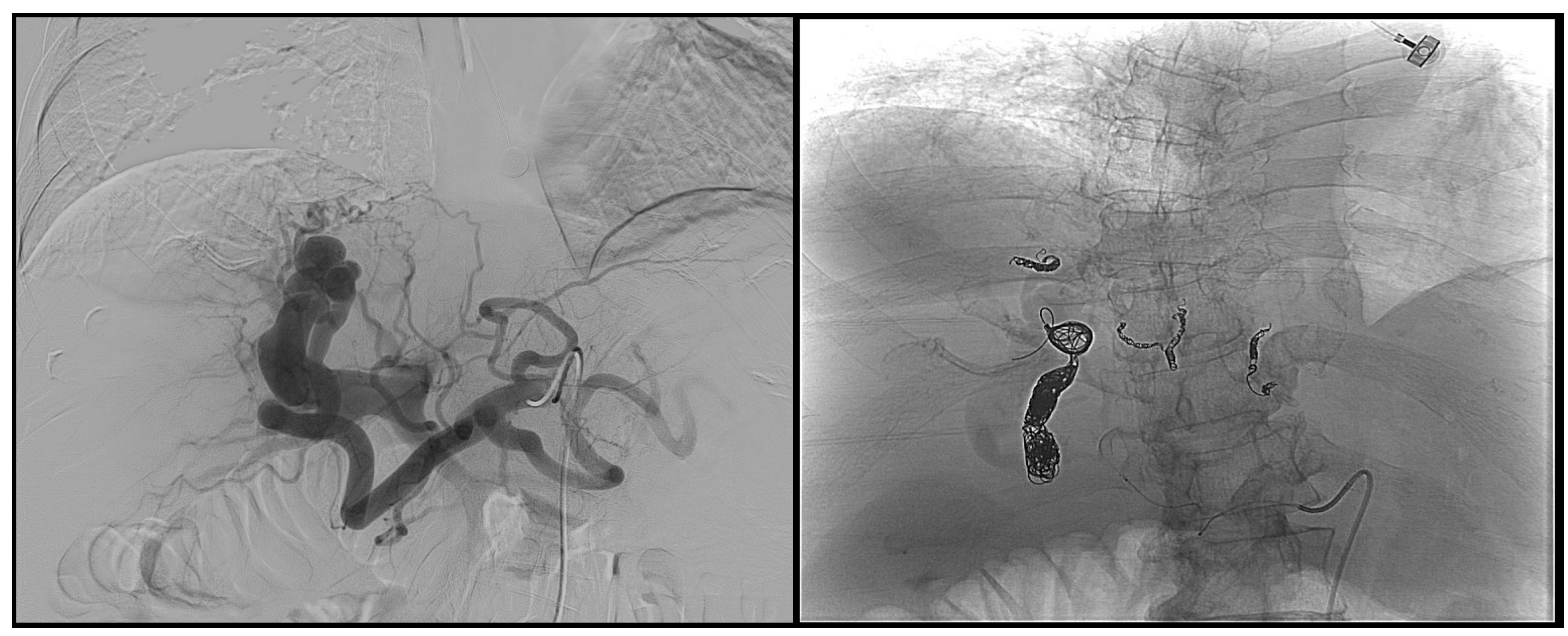

Discussion

- Non-cirrhotic portal hypertension can be classified as pre-hepatic, intrahepatic, and post-hepatic in origin.

- APFs are a rare cause of pre-hepatic non-cirrhotic portal hypertension, and they can be either congenital or acquired.

- Acquired etiologies include penetrating hepatic trauma, splanchnic artery aneurysms, surgical complications, or they may occur spontaneously in the setting of cirrhosis or liver tumors.

- Large, more central APFs are most likely to present with portal hypertension.

- Small APFs can occur following percutaneous liver biopsies, but large APFs complicating a remote percutaneous biopsy, as in our case, are rare.

- Congenital APFs are intrahepatic and diffuse, and typically present in children. However, there are some case reports of adult onset congenital APFs in the literature.

- APF should be considered in patients with noncirrhotic portal hypertension and, when identified treatment is embolization or surgery, not TIPS.

\section{References}

Guzman et al. Arterioportal fistulas: introduction of a novel classification with therapeutic implications. Journal of Gastrointestinal Surgery 2006 Apr;10(4):543-50.

Johnson, et al. Endovascular embolization of large hepatic AV fistulas. Seminars in Interventional Radiology 2007; Mar;24(1): 87-95.

Zhang DY, et al. Portal hypertension induced by congenital hepatic arterioportal fistula: report of four clinical cases and review of the literature. World Journal of Gastroenterology 2015; Feb 21;21(7):2229-35.

Nookala, et al. Isolated arterioportal fistula presenting with variceal hemorrhage. World Journal of Gastroenterology 2013; May $7 ; 19(17): 2714-7$ 